\title{
Failure in chain link securing a round cargo on heavy vehicle
}

\begin{abstract}
Cargo is transported on trucks and trailers all over the country every day. Cargo that is carelessly secured might get damaged, or if it shifts in transit can cause injury or road accidents. Correctly securing the cargo to the vehicle ensures its safe arrival at its destination. This paper describes an incident of chain-link failure which caused a fatal road accident. In this incident, a ten ton metal sheets rolled lashed to a truck came loose when a chain failed during an emergency brake. The metal sheets rolled, rolled toward the cab, crushing and killing the driver. The purpose of this study is to describe the chain-link failure investigation, to pin-point the cause of the chain-link failure and to increase the attention to the aspect of correctly secure round cargo to heavy vehicles. Metallographic tests were conducted using a scanning electron microscope (SEM) to define the chain-link failure areas. Analysis of the results indicates that chain-link failure is predominantly caused by problem in the operational environment, i.e. fault caused by incorrect lashing - placing the chain-link over a sharp corner.
\end{abstract}

Keywords: chain, chain-link failure, road accident, cargo, cargo restraint, heavy vehicles, road transport, trailer
Volume 4 Issue 3 - 2017

\author{
Tsadok Tsach, Finkelstein Nir, Nikolai Volkov \\ Division of Identification and Forensic Science, Israel Police \\ Headquarters, Israel \\ Correspondence: Nir Finkelstein, Scientific Officer, Division \\ of Identification and Forensic Science, Toolmarks and materials \\ Laboratory, Israel Police Headquarters, I Haim Bar-Lev Road. \\ Jerusalem, 9190600 , Israel, Tel 972506275573, \\ Email nirfi7@gmail.com
}

Received: March 13,2017| Published: March 16, 2017

\section{Introduction}

Cargo is transported on trucks and trailers all over the country every day. Cargo that is carelessly secured might get damaged, or if it shifts in transit can cause injury or road accidents. Correctly securing the cargo to the vehicle ensures its safe arrival at its destination. It is estimated that about $25 \%$ of accidents involving trucks are caused by improper loads fastening. ${ }^{1}$ Although suitably trained and skilled workers are required to load and unload cargo, responsibility for restraining the cargo to the vehicle and ensuring that it remains fast throughout the trip should lie with the driver. The driver must be responsible for checking the restraints including the fastening fittings, regardless of whether he or she was involved in restraining the cargo or not. The driver must be familiar with the effect of the cargo on the vehicle and on its stability, and must also be aware of the effect of extreme conditions, such as sudden braking when driving at high speeds, sudden swerving, or crashing. The driver must check the cargo restraints after performing a sudden maneuver such as braking at high speed or swerving. This paper describes an incident of chain link failure which caused a fatal road accident. The purpose of this study is to describe the chain link failure investigation, to pin-point the cause of the chain link failure and to increase the attention to the aspect of correctly secure round cargo on heavy vehicles. To transport metal sheets rolled fast and safe without damaging it, it is necessary that the metal sheets rolled has to be in stable condition. ${ }^{2}$ Lashing chains are mostly used. Lashing chains for cargo on trailers or trucks are constructed of interlaced metal links; each link is made of a bent carbon-steel rod that is laced through the link before it. Restraint failure can occur due to poor design, manufacturing flaws, incorrect use, or inappropriate maintenance. In order to investigate the chain failure, it is important to understand the chain link manufacturing process. During manufacture, an electrical arc is used to join the ends of the bent rod to make a closed link. The join is made by applying pressure in the center of the link so that both ends of the link are forced together. To make them resistant to wear they are thermally treated in an induction oven to a temperature of approximately 800 degrees centigrade, and then quenched in water. Quenching is a continuous process. The next step in chain manufacture is stress relieving the chain at a lower temperature (ca. 500 degrees centigrade). The chain quality rating is embossed on its links: a one digit signature on every 20th link or one link every meter. Quality rating, called grade, ranges between 3 and 8. A larger number indicates greater chain quality and greater tensile strength. During the last stage of manufacturing, the chain is load tested in its elastic range. For this test, grade 4 links are loadtested at double their working load, and grade 8 at X2.5 their working load. Once the load has been released, the chain is expected to return to its original state with no distortion. To ensure chain quality and safety, the manufacturer conducts destructive testing to test material quality, working load, and manufacturing process. Required routine testing before use includes a thorough link-by-link visual inspection, a general inspection of chain structure and end fittings to discover deformations, breaks, cracks, severe corrosion, exposure to higher than permissible heat, chain elongation (by 3\%-5\%) and erosion (of $10 \%$ ) of the chain diameter. If any of these is found the chain must be immediately rejected..$^{3,4}$

When lashing a cargo to a vehicle, the cargo's geometric shape, size, weight, and volume must be taken into account. Cargo with small or large parts, rods, cylinders, coils, plates, etc. require different methods of lashing. When lashing cylindrical cargo to a trailer, wood or rubber wedges must be placed across it, their longitudinal axis perpendicular to the vehicle's length, to prevent rolling (the object will tend to roll toward the front or the back of the vehicle). To overcome the risk of a metal cylinder breaking through the cab wall if the driver brakes suddenly, the cargo must be prevented from shifting by making it a single unit with the vehicle or trailer. The cargo must be lashed using a tie -down system, preferably a chain, and anchored to the lashing points on the carrying vehicle (Figure 1). 


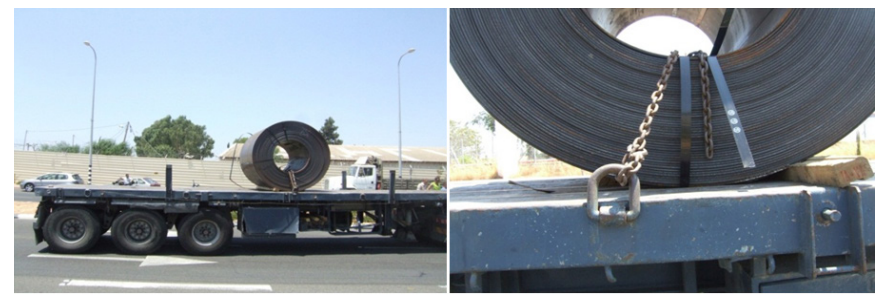

Figure I General view of a trailer carrying a metal sheets rolled.

If the cargo is not properly tied down to the vehicle, the forces acting on the cargo and vehicle will change when there is a change in the direction of movement (inducing circular motion) or when slowing down or accelerating (inducing vertical forces). Without lashing, friction isn't strong enough to prevent the cargo from shifting when the vehicle slows down or accelerates. So, when deceleration is greater, the cargo will continue to move proportionally in the original direction of motion. This is because in this case, the sum of forces between the cargo and the vehicle is not zero $(\Sigma \mathrm{F}=\mathrm{MA} \neq 0)$; in other words, the cargo is in motion relative to the vehicle. For larger masses there is a greater chance that the cargo will shift. During sudden braking at high speed, i.e. deceleration, the forward force equals the product of the cargo mass and the static friction coefficient. Once the cargo is in motion, the friction coefficient changes from static to dynamic; the dynamic coefficient is smaller than the static coefficient, so it will take a greater force to stop the cargo. During transit, the forces developing in the direction of travel can reach $80 \%$ of the cargo weight, and are a function of the friction coefficient and acceleration/ deceleration. Friction, as expressed in the friction coefficient, is also affected by the type of materials and nature of the surfaces. ${ }^{5}$

\section{Case study}

A 10-ton metal sheets rolled was loaded onto a trailer and lashed to the trailer sides using a chain tensioner, similar to Figure 1. Chain link diameter was $10 \mathrm{~mm}$. To prevent the cargo from shifting during transport, wooden bars were placed right up against the metal sheets rolled. During the ride, the truck approached a traffic light at a speed of $50 \mathrm{~km} /$ hour (according to the tachometer); the light then changed to red and the truck driver braked so that the truck would stop in front of the white line (which defines the junction area). The force of deceleration broke the chain, so the cargo advanced toward the cab and hit the driver (Figure 2). On its way the cargo crushed the truck wall as well as the cab (Figure 3), it then fell on to the road and continued to roll several meters until it was stopped by a street pole.

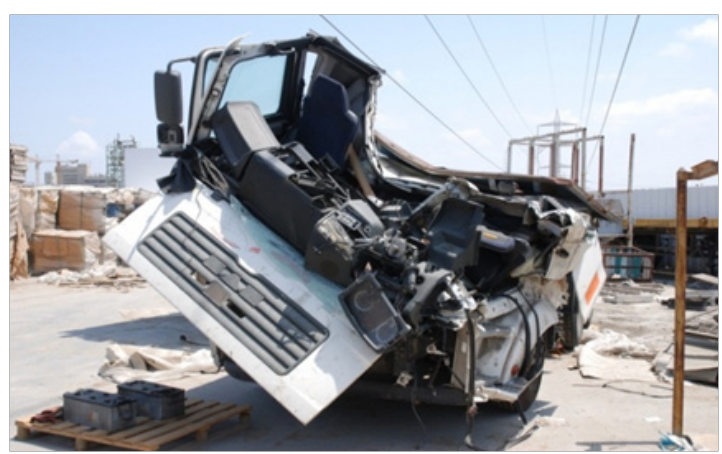

Figure 2 The truck after the crash.

Several exhibits were collected from the crash site:

i. Two segments of metal chain with $10 \mathrm{~mm}$ thick links, from the chain that lashed the cargo to the trailer. One segment was $40 \mathrm{~cm}$ long with a forged 5/16" $9 \mathrm{~cm}$ hook (Figure 4a). The second piece was $250 \mathrm{~cm}$ long and had no lashing fitting on it (Figure 4b).

ii. A link that had connected the two chain segments. The link was open and distorted (Figure 5).

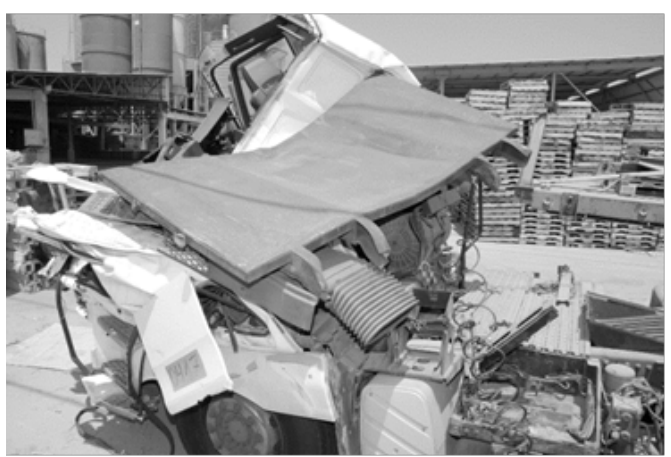

Figure 3 Close-up of the warped wall. Distortions in wall dividing the trailer from the cab.

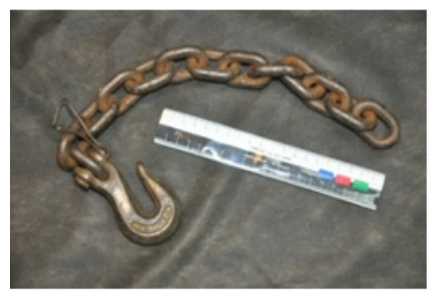

A

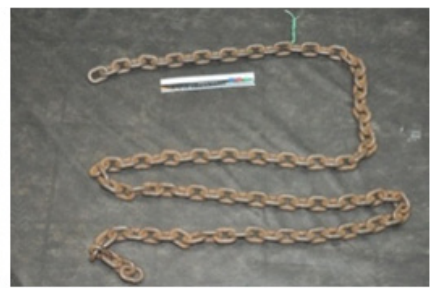

B
Figure 4 Chain segments ( $A$ and $B)$.

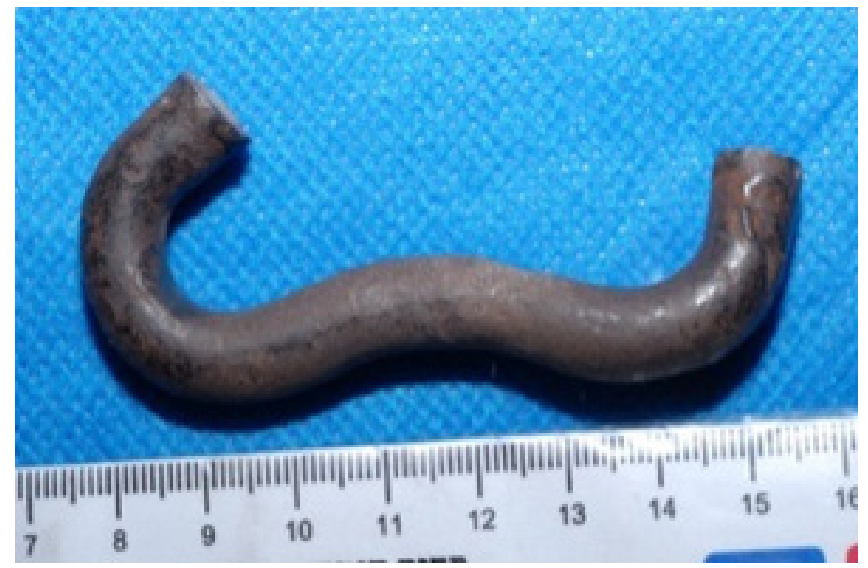

Figure 5 And the opened link.

The accident investigator brought the exhibits to the tool marks and materials laboratory in the division of identification and forensic science in order to determine the cause of the chain failure. When investigating such events, the expert examined the surface of the link to identify signs of fracture and analyze its stages of development. First the type of fracture is identified: impact, overload, or fatigue fracture following long-term wear at lower stresses than that part's maximum strength. The expert also determined the maximum strength of the chain's weakest closed link. Metallographic tests were conducted using a scanning electron microscope (SEM) to examine the surface of the link. Several cracked and visibly un-cracked links were tension tested on an Instron KPX instrument to examine the maximum strength. 


\section{Results and discussions}

The metallographic examination of the failed link, connecting the two parts of the chain, showed plastic deformation in its center (Figure 4). The link diameter was narrowed and a bottleneck was formed on it as a result of tensile forces exerted beyond its yield point. Plastic deformation produced a reduced diameter of $8.80 \mathrm{~mm}$, instead of $10 \mathrm{~mm}$, which is a $12 \%$ difference. As a result of the plastic distortion developed, the link broke, opened and the two parts of the chain separated. Fatigue fractures generally have an area in which the initial crack formed; the fracture then develops further smooth surfaces around the original crack. A morphological examination of the fracture surface on the failed link showed various areas as shown in Figure 6.

a) Figure 6 zone A shows a planar area with cleavage in the center of the cross-section, compatible with a brittle fracture caused by overload. This area constitutes approximately $20 \%$ of the link's cross-section. The chain was quenched during manufacture creating created an area with improved erosion resistance; this means that during overload a fracture would present brittle material properties. Cleavage occurred during the last stage of the link breaking, over a short period of time. The images in Figure 7 were taken in the cleavage area of the open link, ${ }^{6}$ at various magnifications. It is possible to discern a brittle fracture mechanism in these images, which is typical of overload.
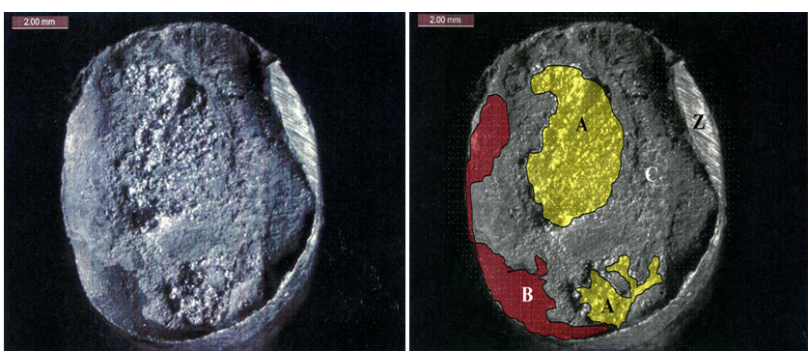

Figure 6 Various areas on the failed link's fracture surface. Crushed zone $(Z)$, break zone $(A)$, pre-failure rusty zone $(B)$, ductile fracture zone $(C)$.
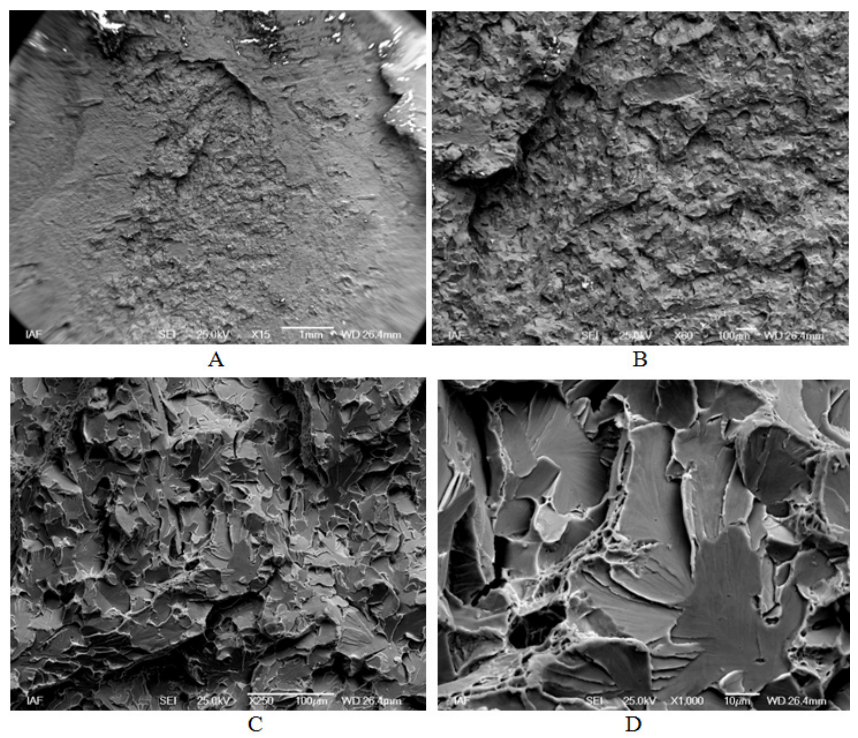

Figure 7 Detail of cleavage (A) caused by overload, magnified xI5, x60, x250, and $x \mid 000$ respectively. b) Figure 6 zone B shows a reddish planar area (rust) over $20 \%$ of the link cross-section. This rust was formed before the break. Rust decreases the effective cross-section of the link, so the actual maximum load that the chain could bear was lower than what it was designed for, in proportion with the change in cross-section surface area. Figure 8 show this area at several magnifications.

c) Figure 6 zone $\mathrm{C}$ shows a planar area with a bumpy surface taking up approximately $60 \%$ of the link cross-section. This bumpy texture is typical of a ductile fracture. In addition, zone $\mathrm{C}$ has typical corrosion-related tear pits; like pitting associated with long-term corrosion. ${ }^{6}$ Figure 9 show the area at several magnifications.

d) Figure 6 zone $\mathrm{Z}$ shows the left edge of the link with the unidirectional crush zone.
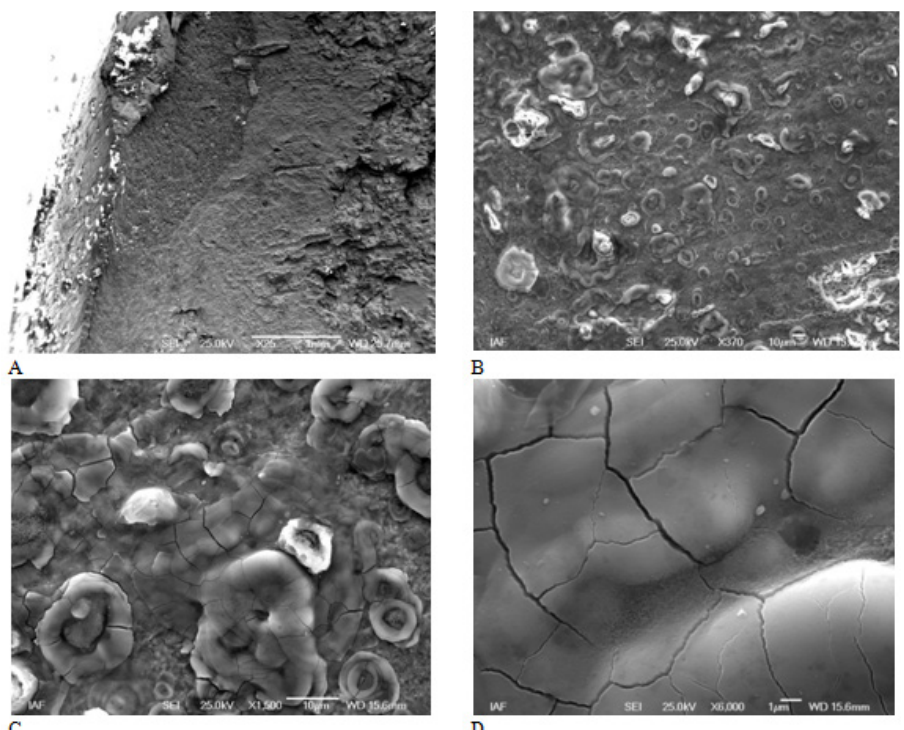

Figure 8 Rusted zone (B) magnified x25, x370, xI500, x6000, respectively.
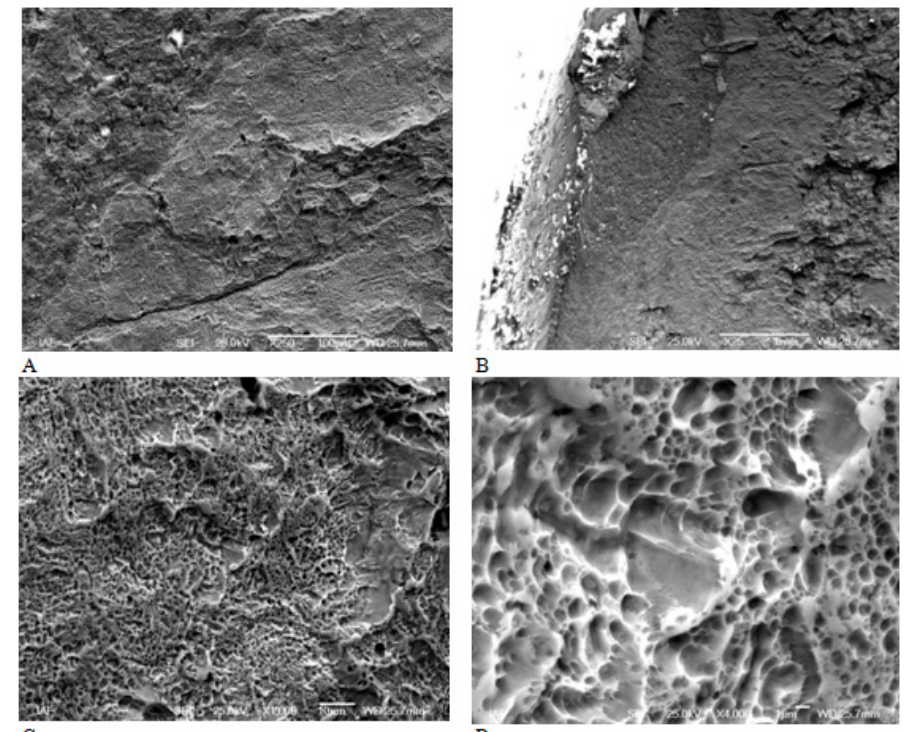

B

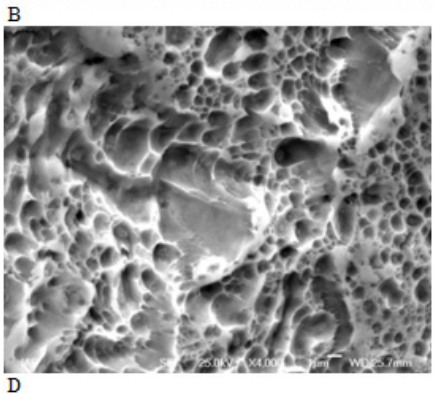

Figure 9 Area $C$ magnified $\times 25, \times 250, \times 1000$, and $\times 4000$, respectively. 
On examining the chain, several more cracked links were found with varying degrees of damage. The link with the largest crack (Figure 10), was tensile tested on an Instron KPX machine. The link collapsed at a 3.8 ton load. Morphology of the fracture surface area on this link, indicated that the link had the same type of zones, although the ratios were different. The most rusted area (Figure 11 zone D) covered close to $70 \%$ of the link's surface; $20 \%$ is cleaved (Figure 11 area $\mathrm{E}$ ), and the remaining area is an overload ductile fracture (Figure 11 area F). To test the chain's strength, several tensile tests were conducted on links that showed no visual signs of cracks on initial inspection. Yield load was 5.5-6 tons. All the tested links failed at their weld point. Morphological examination of the fracture surface on these links, showed that some of the links were not rusted. Some had the three areas described earlier, with different ratios. The rusted area takes up less than $10 \%$ of the cross-section (Figure 12 area G). When examining the results in view of the theory, it is very evident that the highest load that can be placed on a link is proportional to the effective cross-section of that link, excluding the rusted surface area. The disconnected link that directly caused the accident was only rusted over $20 \%$ of its cross-section, this was compared to the most rusted link tested (Figures 10\&11) in the lab which was rusted over approximately $70 \%$ of its cross-section. The conclusion is that the failed link was exposed to additional force to the tensile force. Otherwise, the failed link would have been the most rusted link (Figures 10\&11).

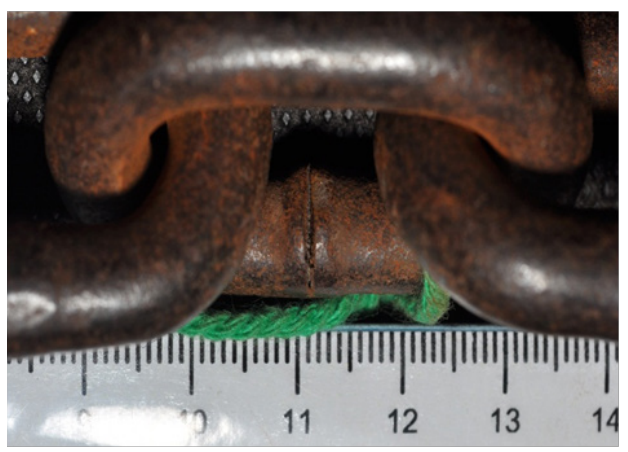

Figure I 0 Another cracked link from the chain.

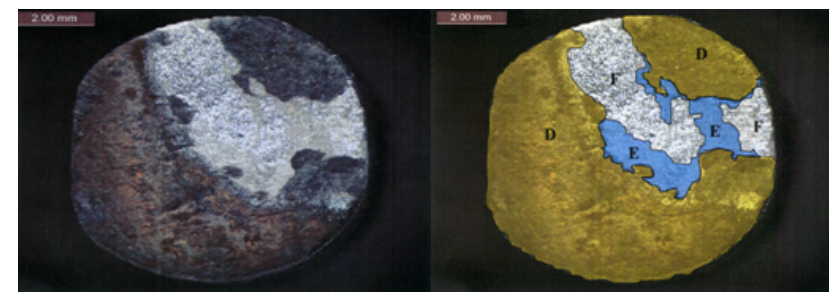

Figure I I Morphology of a cracked link from the same chain.
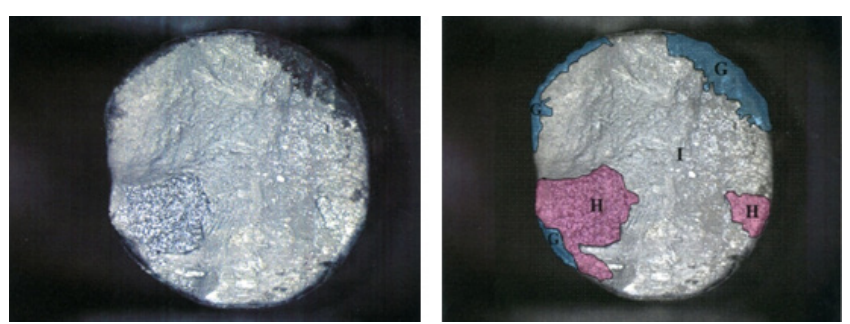

Figure 12 A link showing signs of minor corrosion (under 10\%).

Looking for the additional force, the disconnected chain segments were measured, a $40 \mathrm{~cm}$ piece was found. This length corresponds with the distance between the point where the chain was anchored to the trailer and the hole of the metal sheets rolled. The failed link was placed directly on the sharp edge of the cargo (Figure 6). When the truck decelerated, before the chain broke, additional force was created in the direction of the sharp edge. When the truck decelerated, the force between the metal cargo and the chain grew, caused a plastic deformation that produced a reduced diameter of $8.80 \mathrm{~mm}$, instead of $10 \mathrm{~mm}$, which is a $12 \%$ difference. As a result, a high stressconcentration area was developed. This force, in addition to the tensile force, caused the failure of the specific link. The link failed because it was placed directly on the edge of the cargo, where there was a sharp edge that constituted a crush zone and a high stress-concentration area (Figures 13\&6 zone Z).

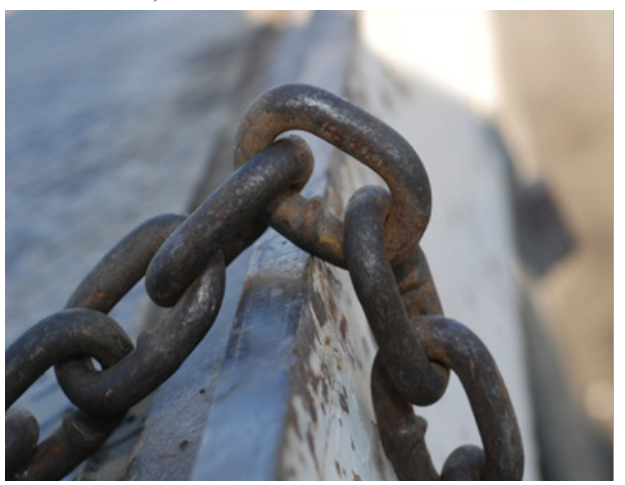

Figure I 3 Method of lashing the cargo so that a stress concentration point is created on the chain (simulation).

\section{Conclusion}

The chain failed for the following reasons:

i. Poor maintenance, resulting in rust which reduced the effective cross section of the link,

ii. Incorrect lashing created a high stress-concentration point on the failed link.

Failure could have been prevented if both the following had occurred: the driver or some other qualified person had inspected the chain before the cargo was lashed, and the contact points between the tie down system and the cargo (mainly for metal cargo) had been inspected to ensure no damage could be incurred to the lashing system through friction on sharp angles or single-point load when braking suddenly. When lashing a sharp-edged cargo, corner shields and protective sleeves must be used wherever indicated. Additional safety measures that can be applied to prevent the cargo shifting during acceleration or deceleration are for example, wooden triangular stoppers (friction coefficient between wood and metal is greater than metal to metal), securing the cargo by lashing it to the lashing surface with an additional chain and shackle.,

\section{Acknowledgments}

None.

\section{Conflicts of interest}

None.

\section{References}

1. European Best Practice Guidelines on Cargo Securing for Road Transport European Commission, Directorate-General for Energy and Transport, USA, pp. 1-208. 
2. Kwac LK, Kim JY, Kim HW, et al. Stability analysis of dunnage for transportation of a steel roll coil. Int J Mod Phys B. 2006;20(2527):3769-3774.

3. BS EN 818-7:2002+A1:2008 Short link chain for lifting purposes. Fine tolerance hoist chain. Grade T (Types T, DAT and DT). British Standards Institution.

4. DIN adopted European Standard. Europe. 2008.
5. Cross WB, Allen RR, Panahshahi N, et al. Analysis of rules and regulations for metal coil truck transport. Journal of transportation engineering. 1996;122(6):475-480.7

6. Victor K, Austin P. Modes of fracture. ASM Handbook. 1987;12:33-79.7

7. Maria C, Grażyna H. The problem of proper cargo securing in road transport-case study. Transport Problems. 2013;8(4):27-33. 